\title{
A review on image enhancement with deep learning approach
}

\author{
Amanjot Kaur $^{1 *}$ and Gagandeep ${ }^{2}$ \\ M.Tech Research Scholar, Department of Computer Science, Punjabi University, Patiala, Punjab ${ }^{1}$ \\ Associate Professor, Department of Computer Science, Punjabi University, Patiala, Punjab ${ }^{2}$
}

(C2018 ACCENTS

\begin{abstract}
Deep learning is presently a dynamic research zone in machine learning and pattern recognition society. It has importance in an expansive zone of applications, for example, speech recognition, natural language processing, and computer vision. The procedures created from deep learning research have just been affecting the exploration of image enhancement. Images caught in low-light conditions more often than not experience the ill effects of low difference, which expands the trouble of consequent PC vision errands in an incredible extent. Many applications mean to upgrade brilliance, differentiate and decrease noisy content from the images in an on-load up constant manner. We demonstrate that a variation of the stacked-inadequate denoising auto encoder can figure out how to adaptively improve and denoise from artificially obscured and disruption included preparing cases.
\end{abstract}

\section{Keywords}

Deep learning, Image enhancement, Image denoising, Auto encoder, Neural networks.

\section{Introduction}

Great quality images and recordings are vital to basic robotized and human-level basic leadership for errands extending from security applications, military missions, way wanting to medicinal diagnostics and business recommender frameworks. Spotless, superior quality images caught by camera frameworks give better proof to all around educated strategy. Be that as it may, cost imperatives constrain extensive scale uses of such frameworks; therefore reasonable sensors are normally utilized. Inadequate lighting, still low sensor quality, deliver images noisy that may hinder Intelligence, surveillance and reconnaissance (ISR) missions and business ventures[1].

Noise is a major issue in estimating light. Regardless of how great the sensors are, there is disruption in images, particularly in low-light conditions or in the dark environment. Image denoising is the procedure of decreasing undesired disruption in images. An image denoising methodology takes a noisy image as input and yields an image where the noise has been decreased. There are many applications remain to profit by changes in image enhancement calculations.

\footnotetext{
*Author for correspondence
}

Recently, deep learning-based methodologies increased huge footing as they are appeared to beat other cutting edge machine learning equipment in numerous computer vision applications, including object detection, image classification and scene understanding. While neural systems have been generally examined for images denoising errands, there are no current works utilizing deep systems to both upgrade and denoise images taken in ineffectively enlightened situations. We approach the issue of low-light images improvement from a portrayal learning point of view utilizing deep auto encoders prepared to pick up basic signals includes in low-light images and adaptively light up and denoise. The strategy exploits nearby way quick complexity change like the works in with the end goal that enhancements are done in respect to nearby neighbours to counteract over amplifing splendid pixels. A similar system is prepared to learn noise structures so as to deliver brighter, denoised images.

\section{Related work}

\subsection{Contrast enhancement}

There are notable contrast enhancement techniques, for example, enhancing image contrast by histogram equalization [2]. Histogram stretching techniques and its variants like brightness preserving bi-histogram equalization(BBHE) and quantized bi-histogram equalization (QBHE) gained prominence to enhance on the artifacts of histogram equalization[3,4]. Along 
these, an optimization technique, OCTM was presented for mapping the contrast-tone of an image with the utilization of mathematical transfer function [5]. Contrast-limiting adaptive histogram equalization (CLAHE) has a place with the class of histogramstretching techniques and serves to point of confinement to the degree of contrast enhancement result of histogram equalization [6]. However, this requires weighting of some area learning as well as an associated complexity increment. Accessible plans additionally investigated utilizing non-linear functions like the gamma function to improve image contrast. A low-light model is proposed and bright channel prior is used to calculate transmission in our low-light model [7]. In [8], LLCNN learns to adaptively enhance image contrast and increase image brightness. A special module is designed to help training and improve the performance. Also find that SSIM loss suits better for low-light image enhancement task. Some comparison on deep learning models shown in Table 1.

Table 1 Comparison on different deep learning models and other metrics

\begin{tabular}{llllll}
\hline Name & Model & Noise level & Patch size & SSIM & PSNR \\
\hline Zhang K et al (2017) & DCNN $^{1}$ & $\sigma=25$ & $35 \times 35$ & 0.819 & 29.02 \\
Lore KG et al (2017) & LLNet $^{2}$ & $\sigma=18$ & $17 x 17$ & 0.92 & 20.35 \\
& S-LLNet & & & 0.87 & 18.07 \\
Tao L et al (2017) & DCNN & - & $41 \times 41$ & 0.846 & - \\
\hline
\end{tabular}

${ }^{1}$ Denoising Convolutional Neural Network (DnCNN)

${ }^{2}$ Low Light Net (LLNet)

${ }^{3}$ Staged Low Light Net(S-LLNet)

\subsection{Image denoising}

Image denoising tasks have been explored using BM3D[9], non-linear filters[10] and KSVD[11]. Using deep learning, authors in [12] exhibited the idea of denoising autoencoders for learning features from noisy images while [13] applied convolutional neural networks to denoise natural images. The network was applied for deblurring and inpainting[14]. Stacked denoising auto encoders were used in[15] to reconstruct clean images from noisy images by exploiting the encoding layer of the multilayer perceptron (MLP). In expansion, authors in[16] implemented an adaptive multi-column architecture to robustly denoise images by training the model with various different kinds of noise and testing on images with arbitrary noise levels and type. In [17] author look over the creation of feed-forward denoising convolutional neural networks (DnCNNs) to grab the progress in very deep architecture, learning algorithm, and regularization procedure into image denoising. Specifically, residual learning and batch normalization are used to speed up the training process as well as boosting up the denoising performance.

\section{Deep learning}

Deep Learning, as a branch of Machine Learning, utilizes calculations to process information and impersonate the reasoning procedure, or to create deliberations [18]. Deep learning (DL) utilizes layers of calculations to process information, comprehend human discourse, and outwardly perceive objects.
Data is gone through each layer, with the yield of the previous layer giving contribution to the following layer. The main layer in a system is known as the info layer, while the latter is called a yield layer. Every one of the layers between the two are mentioned to as hidden layers as appeared in the Figure 1. Each layer is commonly a straightforward, uniform calculation containing one sort of actuation work. Learning can be directed, unsupervised or hybrid.

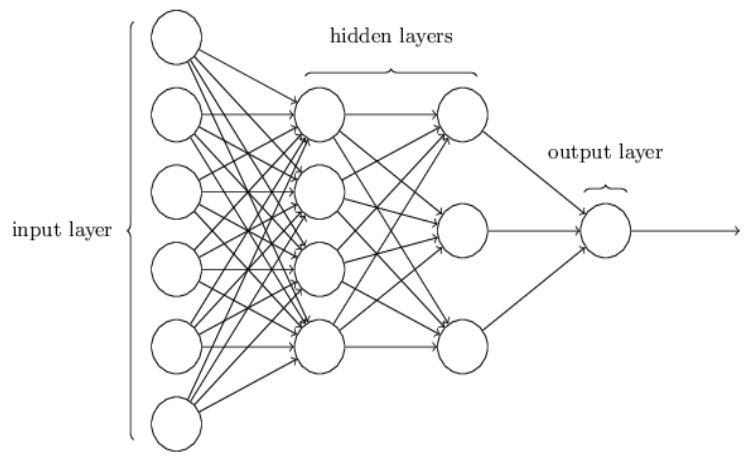

Figure 1 A feed-forward neural network

\section{Image enhancement}

Image enhancement is the way toward altering computerized images with the goal that the outcomes are more reasonable for show or further image investigation. Image enhancement should be possible by evacuating noise, image sharpening, or lighting up an image, making it less demanding to distinguish the 
key highlights[19]. Image enhancement algorithms include deblurring, filtering, and contrast methods. Deep learning utilizes neural systems to learn helpful portrayals of highlights straightforwardly from image information. Neural systems are pretrained to distinguish and remove different sorts of disruption from images and improve the images.

\subsection{Methods in image enhancement}

There are certain methods for Image Enhancement some of them are listed below [1]:

- Histogram matching: Histogram matching is the change of a images with the goal that its histogram coordinates a predefined histogram. The surely understood histogram equalization strategy is an exceptional case in which the predetermined histogram is consistently appropriated.

- Contrast-limited adaptive histogram equalization (CLAHE): It is used to enhance the contrast of the grayscale image assumed as I by transforming. CLAHE works on small regions in the image called tiles, rather than the whole images[1]. Contrast of every tile is enhanced, therefore, the histogram of the output region approximately matches the histogram predefined the 'Distribution' parameter.

- Wiener filter: Wiener filter is a filter used to create a gauge of a coveted or target arbitrary process by linear time-invariant (LTI) filtering of an observed noisy process, accepting known stationary signal and noise spectra, and added substance noise. The Wiener filter limits the mean square error between the evaluated random process and the desired procedure.

- Median filter: The median filter is a nonlinear computerized filtering method, regularly used to expel noise from a image. Such noise reduction is a common pre-processing step to enhance the results of later processing for example, edge recognition on an image. Median filtering is broadly utilized as a part of digital image processing in light of the fact that, under specific conditions, it preserves edges while removing noise.

- Linear contrast adjustment: In this the contrast adjustment block changes the contrast of an image by linearly scaling the pixel values amongst lower and upper limits. Pixel values that are below or above this range are saturated to the lower or upper limit value, individually.

- Unsharp mask filtering: Unsharp masking (USM) is an image sharpening method, frequently accessible in digital image processing software. The "unsharp" of the name gets from the way that the procedure utilizes an obscured, or "unsharp", negative image to make a mask of the original image. The unsharped mask is then joined with the positive (original) image, constructing an image that is less blurred than the original. The subsequent image, in spite of the fact that clearer, might be a less precise portrayal of the image's subject.

- Deep neural network: Execute image processing undertakings, for example, removing noise from images and constructing high-resolution images from low-resolutions images, utilizing convolutional neural networks. Deep learning utilizes neural networks to learn valuable portrayals of highlights straightforwardly from information. For instance, you can utilize a pertained neural network to recognize the images and remove various type of noise from images.

\subsection{Types of noise}

Gaussian noise: It is statistical noise having a probability density function (PDF) equal to that of the normal distribution, which is also known as the Gaussian distribution[20]. In other words, the values that the noise can take on are Gaussian-distributed. The probability density function $p$ of a Gaussian random variable $z$ is given in (1):

$p(\mathrm{~g})=\frac{1}{\sigma \sqrt{2 \pi}} e^{-(z-\mu) / 2 \sigma^{2}}$

Where $z$ represents the gray level, $\mu$ the mean value and $\sigma$ the standard deviation.

Salt and pepper noise: is a form of noise sometimes seen on images. It is also known as impulse noise[20]. This noise can be caused by sharp and sudden disturbances in the image signal. It presents itself as sparsely occurring white and black pixels. The Probability density function of a salt and pepper noise is given in (2):

$p(z)=\left\{\begin{array}{c}\mathrm{Pa} \text { for } \mathrm{z}=\mathrm{a} \\ \mathrm{Pb} \text { for } \mathrm{z}=\mathrm{b} \\ 0, \text { otherwise }\end{array}\right.$

Where, $z$ represents the gray level, a represents the bright region and $\mathrm{b}$ represents the dark region.

Poisson noise: The appearance of this noise is seen due to the statistical nature of electromagnetic waves such as X-rays, visible lights and gamma rays. The $\mathrm{x}-$ ray and gamma ray sources emitted number of photons per unit time [20]. These rays are injected in patient's body from its source, in medical $x$ rays and gamma rays imaging systems. These sources are 
having random fluctuation of photons. Result gathered image has spatial and temporal randomness. This noise is also called as quantum (photon) noise or shot noise. This noise obeys the Poisson distribution and is given in (3):

$p(\mathrm{k})=\frac{\lambda^{k} i e^{-\lambda}}{k !}$

Where, $\mathrm{k}$ takes value $0,1,2 . ., \lambda$ represents the average no. of events per interval, $\mathrm{e}=2.71$ and $\mathrm{k}$ ! is the factorial of $\mathrm{k}$.

\subsection{Challenges in image enhancement}

Image enhancement itself is a major test in image handling condition. An image might be low quality since its difference is low, or it is noisy, or it is obscured. Numerous calculations have been figured to evacuate these corruptions. The test is the way to evacuate corruptions without influencing the signal [21]. Noise decrease calculations include nearby averaging or smoothing which will blur the edges in the images. Noise levels are hard to predict. Therefore, removing such nosies is a challenging task.

\section{Conclusion and future work}

Deep Learning has emerged as a new field of machine learning, and has obtained extensive interests in different research area. In contrast to traditional methods for image enhancement, Deep Learning has great advantage of inherently providing success in denoising the image. Still deep learning algorithms need to be trained more to get more accurate results. Some possible improvements and future research directions are to train the models with the combination of different noise types that are robust and adaptive in the low light situations such as dusty and foggy sights. Training with poisson noise to simulate more realistic scenarios.

\section{Acknowledgment}

None.

\section{Conflicts of interest}

The authors have no conflicts of interest to declare.

\section{References}

[1] Lore KG, Akintayo A, Sarkar S. LLNet: a deep autoencoder approach to natural low-light image enhancement. Pattern Recognition. 2017; 61:650-62.

[2] Trahanias PE, Venetsanopoulos AN. Color image enhancement through 3-D histogram equalization. In international conference on image, speech and signal analysis 1992 (pp. 545-8). IEEE.
[3] Krutsch R, Tenorio D. Histogram Equalization. Free Semicond Inc.

a. http://cache.freescale.com/files/dsp/doc/app_note/AN4 318.pdf. Accessed 26 March 2018.

[4] Kaur M, Kaur J, Kaur J. Survey of contrast enhancement techniques based on histogram equalization. International Journal of Advanced Computer Science and Applications. 2011; 2(7):13741.

[5] Wu X. A linear programming approach for optimal contrast-tone mapping. IEEE Transactions on Image Processing. 2011; 20(5):1262-72.

[6] Pisano ED, Zong S, Hemminger BM, DeLuca M, Johnston RE, Muller K, et al. Contrast limited adaptive histogram equalization image processing to improve the detection of simulated spiculations in dense mammograms. Journal of Digital Imaging. 1998; 11(4):193-200.

[7] Tao L, Zhu C, Song J, Lu T, Jia H, Xie X. Low-light image enhancement using $\mathrm{CNN}$ and bright channel prior. In international conference on image processing 2017 (pp. 3215-9). IEEE.

[8] Tao L, Zhu C, Xiang G, Li Y, Jia H, Xie X. LLCNN: a convolutional neural network for low-light image enhancement. In visual communications and image processing 2017 (pp. 1-4). IEEE.

[9] Dabov K, Foi A, Katkovnik V, Egiazarian K. BM3D image denoising with shape-adaptive principal component analysis. In signal processing with adaptive sparse structured representations 2009.

[10] Chan RH, Ho CW, Nikolova M. Salt-and-pepper noise removal by median-type noise detectors and detailpreserving regularization. IEEE Transactions on Image Processing. 2005; 14(10):1479-85.

[11] Elad M, Aharon M. Image denoising via sparse and redundant representations over learned dictionaries. IEEE Transactions on Image Processing. 2006; 15(12):3736-45.

[12] Vincent P, Larochelle H, Bengio Y, Manzagol PA. Extracting and composing robust features with denoising autoencoders. In proceedings of the international conference on machine learning 2008 (pp. 1096-103). ACM.

[13] Jain V, Seung S. Natural image denoising with convolutional networks. In advances in neural information processing systems 2009 (pp. 769-76).

[14] Xie J, Xu L, Chen E. Image denoising and inpainting with deep neural networks. In advances in neural information processing systems 2012 (pp. 341-9).

[15] Burger HC, Schuler CJ, Harmeling S. Image denoising: can plain neural networks compete with BM3D? In conference on computer vision and pattern recognition 2012 (pp. 2392-9). IEEE.

[16] Agostinelli F, Anderson MR, Lee H. Adaptive multicolumn deep neural networks with application to robust image denoising. In advances in neural information processing systems 2013 (pp. 1493-501).

[17] Zhang K, Zuo W, Chen Y, Meng D, Zhang L. Beyond a Gaussian denoiser: Residual learning of deep CNN 
Kaur et al.

for image denoising. IEEE Transactions on Image Processing. 2017; 26(7):3142-55.

[18] Chen XW, Lin X. Big data deep learning: challenges and perspectives. IEEE Access. 2014; 2:514-25.

[19] Cheng HD, Shi XJ. A simple and effective histogram equalization approach to image enhancement. Digital Signal Processing. 2004; 14(2):158-70.

[20] Boyat AK, Joshi BK. A review paper: noise models in digital image processing. Signal \& Image Processing: An International Journal. 2015; 6(2): 63-75.

[21] Huang TS, Aizawa K. Image processing: some challenging problems. Proceedings of the National Academy of Sciences. 1993; 90(21):9766-9.

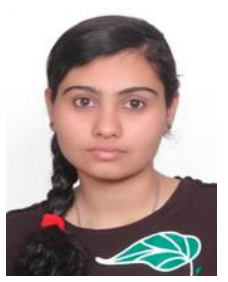

Amanjot Kaur received the B.Tech degree in Computer Science and Engineering from MIMIT Malout, Punjab with $76 \%$ in 2016. She is Currently pursuing M.Tech from Punjabi University, Patiala. Her area of research is Image enhancement in Deep Learning.

Email: amanjotchaha194@gmail.com

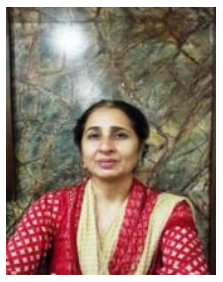

Dr. Gagandeep received her Ph.D degree in Computer Science from Punjabi University, Patiala in 2012 on the topic "Analysis and Development of Testing Techniques for Componentbased Software Systems". Currently, she is working as Associate Professor in the Department of Computer Science, Punjabi University, Patiala. She has more than 20 years of teaching and research experience. She is currently involved in research work on data security, virtualization techniques and computational offloading mechanism for cloud and mobile computing. 